\title{
Performance Evaluation and Comparison of Scheduling Algorithms on 5G Networks using Network Simulator
}

\author{
D. Perdana, A. N. Sanyoto, Y. G. Bisono
}

\author{
Doan Perdana* \\ School of Electrical Engineering \\ Telkom University, Bandung, Indonesia \\ *Corresponding author: doanperdana@telkomuniversity.ac.id
}

\author{
Aji Nur Sanyoto \\ School of Electrical Engineering \\ Telkom University, Bandung, Indonesia \\ ajinsanyoto@gmail.com
}

Yoseph Gustommy Bisono

School of Electrical Engineering

Telkom University, Bandung, Indonesia

bisono@telkomuniversity.ac.id

\begin{abstract}
In this research, we compared the Round Robin (RR) and the Proportional Fair (PF) algorithms for different user equipment density scenarios using voice and video traffic, to evaluate the key impact on performance of $5 \mathrm{G}$ mmwave network. This research simulated on NS3.27 with an integrated mmwave module. Based on the result, we found that the RR is a good choice for voice traffic. It has a throughput of $3.65 \%$ better than $\mathrm{PF}$ with similar fairness index. On the other hand, we found that the $\mathrm{PF}$ is the right choice for video traffic due to has better result for throughput. It has a throughput of $1.24 \%$ better than RR. For fairness index round robin has better result for voice and video traffic.

Keywords: Network simulation, 5G networks, scheduling algorithm, round robin, proportional fair.
\end{abstract}

\section{Introduction}

Increase of internet users give challenge for Information and Technology (IT) industry especially service providers in order to provide high quality and low latency service quality. According to the situation above, the telecommunications industry began to move to fifth generation technology (5G). Millimeter waves that have a frequency spectrum of $28 \mathrm{GHz}-30 \mathrm{GHz}$ appear as a central technology in fifth generation technology $(5 \mathrm{G})$, because of their potential with wide bandwidth to achieve the large throughput required by future networks [20]. It has been proposed to be an important part of the $5 \mathrm{G}$ network to provide multi-gigabit communication services [17]. Research about mmwave generally uses $28-30 \mathrm{GHz}$, free-license bands at $60 \mathrm{GHz}$, and E-bands at 71-76 GHz, 81-86 GHz, and 92-95 GHz. [4]. Mid-infrared ELT Imaginer and Spectrograph (METIS) 2020 provides requirements for 5G technology to have end-to-end (E2E) latency below $10 \mathrm{~ms}$, this cannot be achieved by previous technology [9]. A lot of developer have already innovate not only on the physical layer, but also on several layers. Data allocation for small packages on TDMA scheduling LTE systems is inefficient because the transmission process is sent at fixed $1 \mathrm{~ms}$ Transmission Time Interval (TTI). Flexible TTI which has a flexible TDMA structure has been proposed in the study [8]. The TTI variable system also has flexibility in scheduling resources, which can handle the characteristics of various networks efficiently. The concept of flexible TDMA is a solution, considering $5 \mathrm{G}$ has various types of services with very 
diverse traffic, ranging from applications, devices, and usage. According to all advantages, this flexible scheme must be handled by the right scheduling algorithm. In this research, we analyzed choice of the scheduler has a significant impact on performance of $5 \mathrm{G}$ mmwave network.

In this research, we analyzed choice of the scheduler has a significant impact on performance of $5 \mathrm{G} \mathrm{mmWave} \mathrm{network.} \mathrm{The} \mathrm{module} \mathrm{to} \mathrm{simulate} \mathrm{scheduler} \mathrm{algorithm} \mathrm{that} \mathrm{has} \mathrm{been} \mathrm{adapted}$ to the flexibe TTI concept has been done by [16] in Network simulator 3. All wireless network protocols have similarities in terms of message scheduling [11].

This paper compare two scheduler algorithms and analyze performance parameters such as delay, throughput, and fairness index. We simulate Round Robin (RR) and Proportional Fair $(\mathrm{PF})$ to find the best performing schedulers that are applied to flexible TTI schemes. We analyze the effect of scheduler on network performance such as delay, throughput, and fairness index. Round Robin (RR) is a scheduler that provides resources for users without considering channel conditions. This is a simple procedure that provides fairness [14]. This algorithm works by rotating the queue process. Each process has the same time allotment that is equal to time quantum (q). If this quantum time runs out, the server will handle the next process. There is a matrics to set user priorities for resource blocks. Expressed with matrix $m$ with user i in resource block $k$ [5]. The value of the user metrics above compared with other user metrics during the system. Users with the largest matrices will be served first.

$$
m_{i, k}=w_{i}(t-T i)
$$

Where notation on the (1) known as :

$w_{i}=$ priority value for every service for user i

$t=$ current time

$T i=$ last time when user i was served

Proportional Fair algorithm has main purpose to balance between throughput and fairness among all the users [1]. Different from the previous algorithm, this algorithm considers the channel conditions in the calculation of the matrics. Then the proportional fair algorithm calculates based on the value of the average data rate and throughput in the previous metrics calculation. There is a matrix to set user priorities for resource blocks. Expressed with matrix m with user i in resource block $\mathrm{k}$ :

$$
m_{i, k}=\frac{d_{i, k}(f)}{R_{i}(f)}
$$

With $R_{i}(f)$ is average throughput of user i computed in subframe $\mathrm{f}$, and $d_{i, k}(f)$ is Achieveable throughput user $\mathrm{k}$ in $\mathrm{m}$ resource block and $\mathrm{f}$ subframe which is a Shannon expression for the channel capacity as

$$
d_{i, k}(f)=\log \left\lfloor 1+S N R_{i, k}(f)\right\rfloor
$$

\section{Related work}

In [17] research has been carried out stating that, with the increase in cellular data demand, $5 \mathrm{G}$ exploited the Internet a large number of variations in the millimeter wave (mmWave) band to increase communication capacity. mmWave itself is suitable for $5 \mathrm{G}$ network devices, depending on the communication characteristics of mmWave capable of overcoming system complexity and design, interference management and spatial reuse, anti-blockage, and dynamics due to mobility. The fundamental difference between mmWave communication and other communication systems, 
namely in terms of opposing high propagation, directivity, and sensitivity to blockages. The characteristics of mmWave communication can be utilized as potential for mmWave communication, including integrated design and systems, interference management, spatial reuse, anti-clogging, and dynamic control. In [14] a TTI-based design analysis was conducted and focused on flexible TTI-based designs, in terms of how well they utilized the allocated radio resources, and found that flexible frame structures exceeded fixed structures in all traffic scenarios discussed, especially for small burst traffic. So it can be concluded that the flexible TTI scheme will be very suitable to be applied on mmWave communication.

In [16] a research has been conducted on the implementation and validation of the mmwave module in NS-3. They redesigned several layers because mmWave will require innovation not only in the physical layer, but also across all layers of the communication protocol stack to fully utilize high throughput, low latency capabilities and maximum performance. In [14] research has been carried out stating that, round robin performance and proportional fair scheduling provide good performance for downlink transmission mode. But for different transmission modes, proportional fair is able to provide good data rates. Although round robbin provides individual data speeds that are better compiled and far from eNodeB, the absolute value of this data speed is not as high as the proportional fair. Therefore proportional fair may still be a good choice.

In the [2] study conducted by B. Barakat et al. it was stated that the growth of wireless traffic and the very high demand for data levels from users encouraged researchers to improve the performance of Long Term Evolution-Advanced (LTE-A). To optimize it, package scheduling is done which is able to distribute radio resources among users to improve network performance and spectrum efficiency. In this case it has been proven that generalized Proportional Fair (GPF) Schedulers are better than conventional other schedulers. Several studies about scheduling algorithm on cellular technology has been done in LTE system. S. Ismail et al [12] have compared of several scheduling algorithms and evaluated in terms of throughput, delay, packet loss, and fairness index on vehicular environment for uplink transmission in LTE networks.

In reference [18] shows that, RR algorithm produces a good fairness index and has a poor throughput and has a delay. In contrast, the MT algorithm has good throughput and bad fairness index. The PF algorithm has increased fairness and throughput but has poor delays due to traffic requirements and channel condition independence. 3LHA has good throughput for P1 and P2 connections but makes P3 connections starving. In contrast, 3LHA requires fairness improvement.

In [13], Mohnish Jha et al. compared Round Robin, Priority Set Scheduler and Proportional Fair scheduler by transmitting real-time voice packages and best effort services with changes in the number of users using NS3.24. The simulation results show that the round robin scheduler is better QoS performance compared to the other two at uplink and downlink. Nevertheless, rarely research about scheduling algorithm on $5 \mathrm{G}$ network. Research about $5 \mathrm{G}$ mmwave can be done to evaluate cross-layer and end-to-end performance. Several studies about scheduling on 5G networks also have been researched before. K. Gomez on research [10], provided a comparative study of a different scheduling disciplines that can be used in future 5G especially on emergency communications for public safety. In addition to proposing a new disciplinary scheduler, simulation results.

\section{Research method}

The simulations on this research were performed on the Network Simulator 3.27 with an additional mmWave module. The mmave module is designed for end-to-end simulations of 3GPP style cellular networks.

Figure 1 shows the flowchart system. After designing the module in the NS3 environment, 


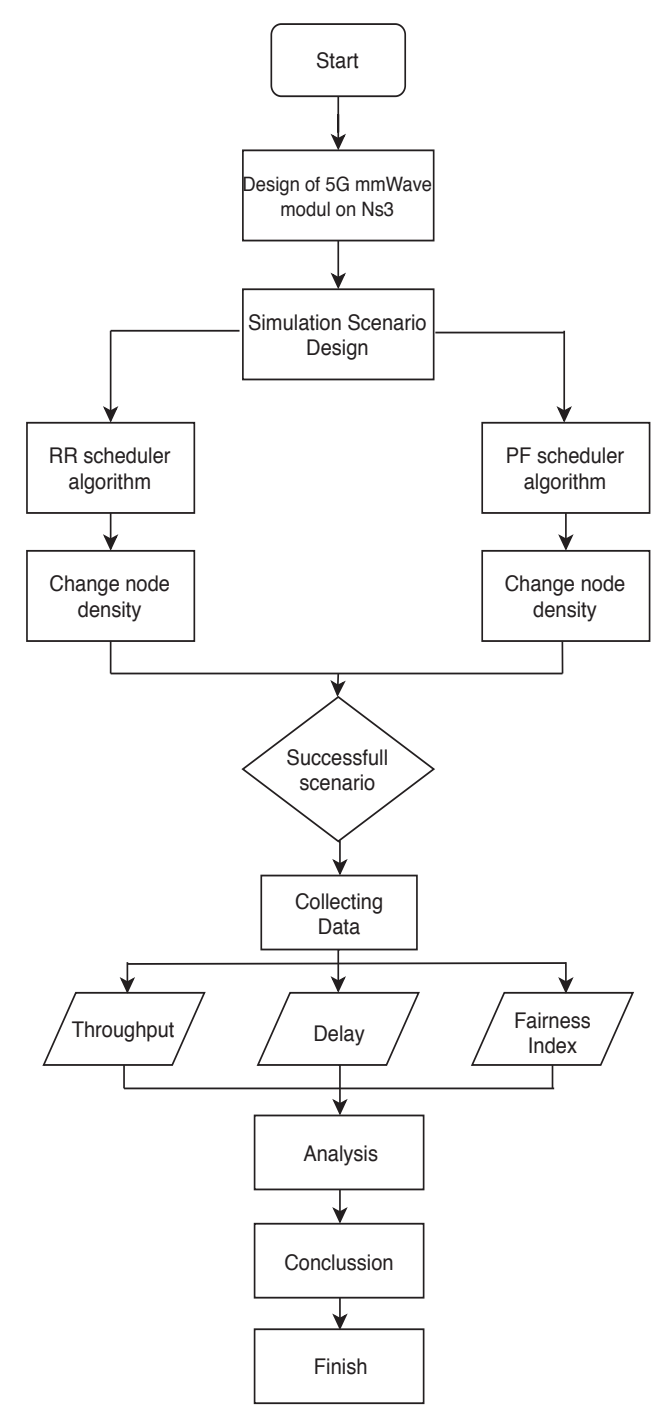

Figure 1: Flowchart system

the simulation design is adjusted to the scenario. The scheduling algorithm is implemented and simulated alternately. Changes on the number of nodes are set gradually from 20,40, 60, 80, and 100 nodes. If the simulation is failed, the simulation scenario design will be reconfigured. If it is successful, we analyzed network performance such as throughput, delay, and fairness index. Throughput defined as the effective ability of a network in sending data. Throughput is the number of packets received in bits divided by the amount of delivery time [21].

$$
\text { Throughput }=\frac{\sum R x \text { Packet Size }}{\text { Delivery Time }}
$$

Delay, defined as the time from packet send from sender to received in destination [19]. Average End to End Delay, which is the average time of delivering the data packet from the sender to the receiver [15]. The delay value starts calculated when the source starts sending packets and ends when the destination actually receives the packet. The delay can be constant, time-varying, or even random depending on the scheduler [3]

$$
\text { Delay }=\frac{T_{r x}-T_{t x}}{\sum R x}
$$


Table 1: Simulation parameters

\begin{tabular}{|c|c|}
\hline Parameter & Quantity \\
\hline Mmwave Carrier frequency & $28 \mathrm{GHz}$ \\
\hline Mmwave Bandwidth & $1 \mathrm{GHz}$ \\
\hline Number of eNodeB & 1 \\
\hline Number of User & $20,40,60,80,100$ \\
\hline User Mobilility & Constant Position \\
\hline Datarate & Voice $:$ Kbps, Video $: 386$ Kbps \\
\hline Packet Size & Voice $: 20$ bytes, Video $: 240$ bytes \\
\hline Transport Layer & UDP \\
\hline Scheduler Algorithm & Round Robin, Proportional Fair \\
\hline
\end{tabular}

Where notation on the (5) known as :

$T_{r x}=$ Time of received packet on destination

$T_{t x}=$ Time of packet send on source

$\sum R x=$ Received packet

Fairness Index defined as the level of fairness of scheduling algorithms in schedule packages and allocation of resources to be sent. The theory and formula regarding the fairness index was revealed by [6]. Metrics of the formula are known as Jain's Fairness Index. Maximum value of this metrics is 1 , where it indicates [7]

$$
f(x)=\frac{\left(\sum_{i=1}^{n} x_{i}\right)^{2}}{n \sum_{i=1}^{n} x_{i}^{2}}
$$

Where notation on the equation (6) known as : $f(x)=$ fairness index

$n=$ number of user

$x=$ Throughput user i
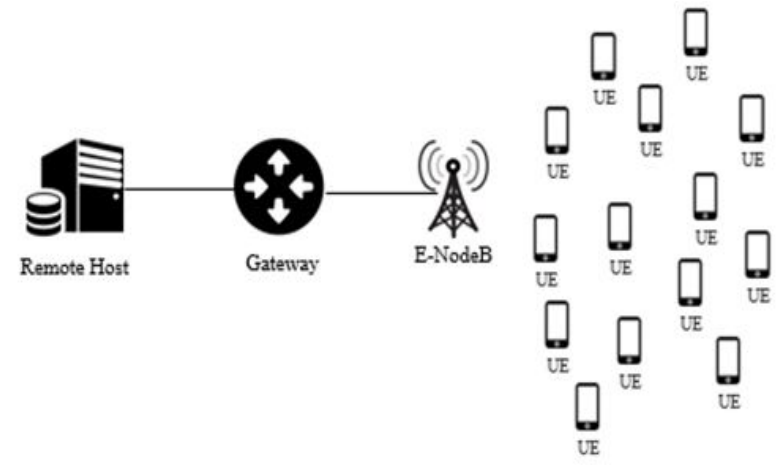

Figure 2: Simulation topology

The system design is shown in Figure 2. The remote host node has a function as the sender and connected to the Packet Gateway (PGW) node in point to point mode. Datarate between PGW and Remote host is $100 \mathrm{Gbps}$. MME has role to control signaling session, PGW was connected to S-GW before eNodeB to send radio transmission using the LTE EPC core network which indicates that the network to be simulated is a non-standalone $5 \mathrm{G}$ network.

The parameters and its description are shown in Table 1 . The scenario in this research is 
to change node density simulated by different scheduling algorithms. Changes in the number of nodes in the scenario vary from twenty to one hundred with intervals of 20 UEs. The scheduler algorithm that will be used is round robin and proportional fair. UEs positions are arranged randomly with constant position mobility model. The simulations generate traffics for voice and video by remote hosts. The number of packet size and data rate used in the simulation is adjusted to the characteristics of the packet size and data rate on one of the VoIP codecs G.729 and H.264 video codec. For G.729 voice codec, which has 8 Kbps data rate and 20 bytes packet size. H.264 video codec which has $386 \mathrm{Kbps}$ data rate and 240 bytes packet size.

\section{Result and analysis}

After simulating voice and video traffic from the 5G mmWave network in NS3, we obtained performance results such as throughput, delay and fairness index, then be analyzed. The analysis divided into two parts for voice and video traffic to find out which better scheduler for the two services.

\subsection{Simulation result for voice traffic}

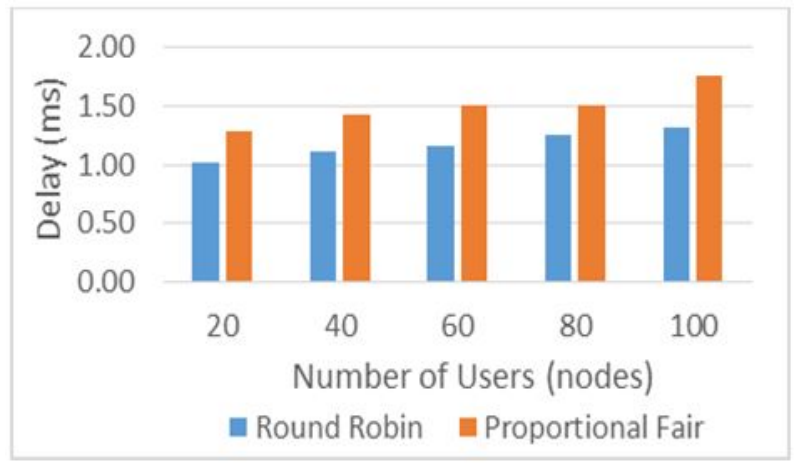

Figure 3: Delay on change number of users for voice traffic

Figure 3 shows the effect when increasing the number of users to the delay obtained from the voice traffic simulation. The lowest delay in round robin occurred on $20 \mathrm{UE}$ with $1.023 \mathrm{~ms}$, for proportional fair lowest delay occurred on $20 \mathrm{UE}$ with $1.285 \mathrm{~ms}$. On $100 \mathrm{UE}$, round robin and proportional fair generating the highest delay with $1.321 \mathrm{~ms}$ and $1.755 \mathrm{~ms}$. Average delay obtained from round robin is $1.215 \mathrm{~ms}$. This is $18.29 \%$ lower than proportional fair with average delay of $1.487 \mathrm{~ms}$. Based on figure 5, it can be conclude that delay for both scheduler increase, due to increase of number of UE make waiting time for each users to be served is getting longer. Round Robin has a better delay because for small packages, users queuing don't to take long time, different with Proportional fair which must take consider the channel quality.

Figure 4 shows effect the when increasing the number of users to the throughput obtained from the voice simulation. It show that, round robin has higher throughput than proportional fair. Round robin gets average throughput of $0.137 \mathrm{Mbps}$. This is $3.65 \%$ better than proportional fair with average throughput of $0.132 \mathrm{Mbps}$. The lowest throughput in proportional fair occurred on $100 \mathrm{UE}$ with $0.131 \mathrm{Mbps}$, and for round robin occurred on $100 \mathrm{UE}$ with $0.132 \mathrm{Mbps}$. It show that the increase number of users, throughput decreased due to the bandwidth capacity will be shared with all users. Round robin has a higher throughput because this algorithm not consider the channel condition and has main purpose to balance between throughput and fairness among all the users. 


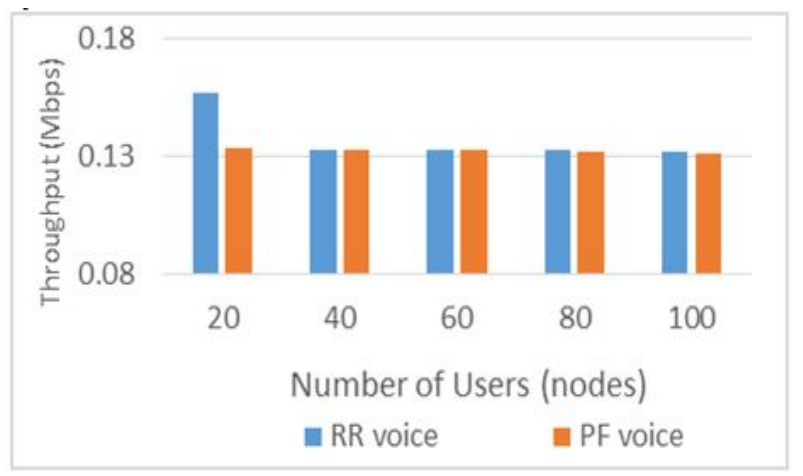

Figure 4: Throughput on change number of users for voice traffic

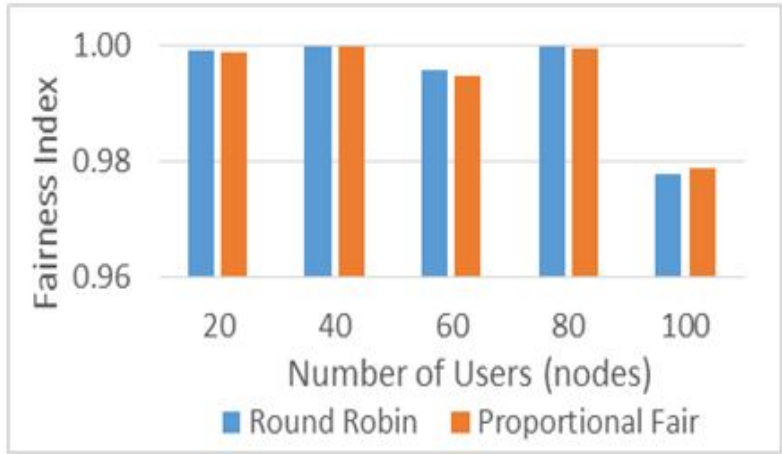

Figure 5: Fairness index on change number of users for voice traffic

Figure 5 shows the effect when increasing the number of users to fairness index in each scheduling algorithm for voice simulation. The average of fairness index obtained from the simulation of adding the number of users to the proportional fair algorithm is 0.994 . It is lower than the round robin's fairness index that has 0.995 . The value obtained by the round robin algorithm is greater because this algorithm does not consider channel conditions so that it offers a higher fairness value. Round robin and proportional fair have a decreasing fairness index value against the increase in the number of users. Its happened because of the increasing number of users, more users were served and reduce the value of fairness. Both schedulers show fairness due to the fairness index close to 1.

\subsection{Simulation result for video traffic}

Figure 6 shows the effect when increasing the number of users to the delay obtained from the video traffic simulation. Average delay obtained from round robin simulation with rising the UE is $3.105 \mathrm{~ms}$. This is $2.19 \%$ higher than proportional fair average delay with $3.037 \mathrm{~ms}$ which make proportional fair has better delay on video traffic. Round robin has higher delay because round robin not consider the channel condition, that make the delay for video traffic. Users served in sequence obtain longer delay.

Figure 7 shows the effect of increasing the users to the throughput obtained from the video traffic simulation. For proportional fair, the highest throughput occurred by 20 users with 2.869 Mbps. The highest throughput in round robin occurred on 20 users with 2.811 Mbps. It shown that round robin and proprotional fair has a decreasing throughput value towards increasing number of user. The average throughput of proportional fair obtained from the simulation is $2.820 \mathrm{Mbps}$. This is $1.24 \%$ higher than round robin with $2.785 \mathrm{Mbps}$. 


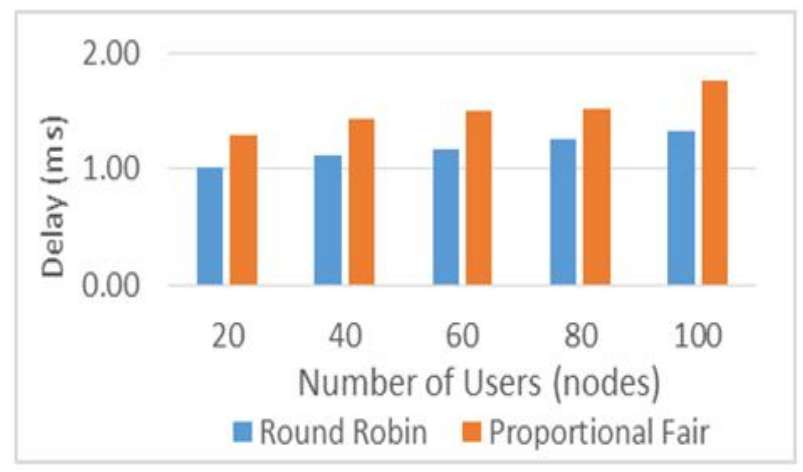

Figure 6: Delay on change number of users for video traffic

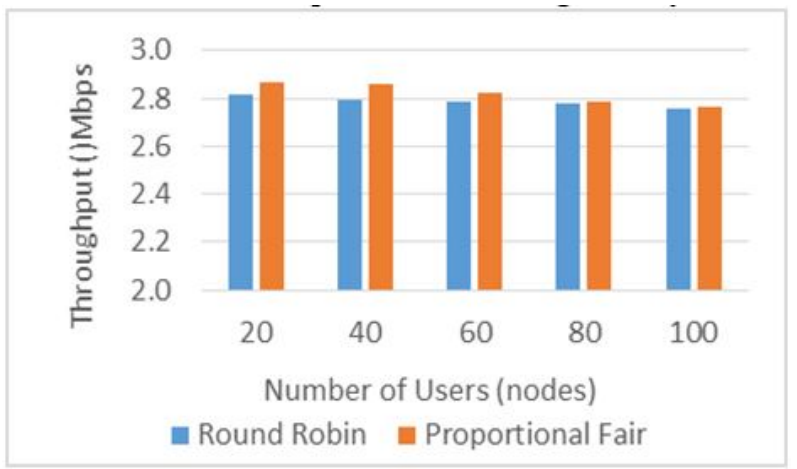

Figure 7: Throughput on change number of users for video traffic

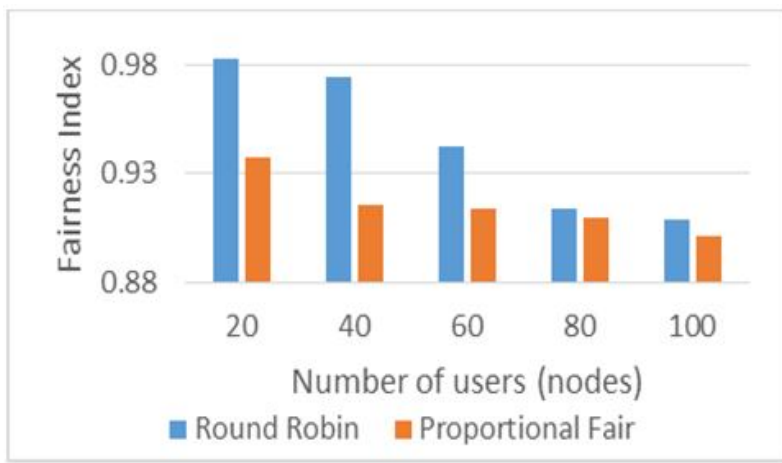

Figure 8: Fairness index on change number of users for video traffic

Figure 8 shows the effect when changing the number of users to fairness index in each scheduling algorithm for video traffic simulation. The average of fairness index obtained from the simulation of adding the number of users to the round robin algorithm is 0.94 . It is $3.19 \%$ better than proportional fair's fairness index that has 0.91 . Round robin and proportional fair have a decreasing fairness index value against the increase in the number of users. Its happened because of the increasing number of users, more users were served and reduce the fairness. Round Robin has higher fairness index than proportional fair because round robin not consider channel condition and prioritize fairness among users. 


\section{Conclusion}

In this paper, our work focuses on scheduling in a $5 \mathrm{G}$ network with a new MAC layer structure that has been proposed in previous studies. Based from simulation result, the choice of scheduling algorithm has affect on network performance. Proportional fair is better than round robin for throughput on video traffic with similar value of fairness index. Round robin has $3.19 \%$ better fairness index than proportional fairness and $2.19 \%$ higher average delay than proportional fair. It can be conclude that proportional fair is the right algorithm for video traffic. Round robin is right choice for voice traffic due to has better result for fairness and throughput. For further research, it is expected that more scheduler algorithms can be implemented in the new design of mac layer on the $5 \mathrm{G}$ network.

\section{Bibliography}

[1] Angri, I.; Mahfoudi, M.; Najid, A.; Bekkali, M. (2018). Exponential MLWDF (EXPMLWDF) Downlink Scheduling Algorithm Evaluated in LTE for High Mobility and Dense Area Scenario, International Journal of Electrical and Computer Engineering (IJECE), 8(3), 1618-1628, 2018.

[2] Aramide, S.O.; Barakat, B.; Wang, Y. et al. (2017). Generalized proportional fair (GPF) scheduler for LTE-A 2017 9th Computer Science and Electronic Engineering (CEEC).

[3] Benitez-Perez, H.; Ortega-Arjona, J. ; Esquivel-Flores, O. et al. (2016). A Fuzzy Networked Control System Following Frequency Transmission Strategy, International Journal of Computers Communication \& Control, 11(1), 11-25, 2016.

[4] Boccardi, F.; Heath, R. W.; Lozano, A. et al. (2014). Five disruptive technology directions for 5G, IEEE Communications Magazine, 52(2), 74-80, 2014.

[5] Capozzi, F.; Piro, G.; Grieco, L. A. et al. (2013). Downlink Packet Scheduling in LTE Cellular Networks: Key Design Issues and a Survey, IEEE Communications Surveys 8 Tutorials, 15(2), 678-700, 2013.

[6] Carpin, M.; Zanella, A.; Rasool, J. et al. (2015). A performance comparison of LTE downlink scheduling algorithms in time and frequency domains, 2015 IEEE International Conference on Communications (ICC), London, 15(4), 3173-3179, 2015.

[7] Donoso, Y.; Lozano-Garzon, C.; Camelo, M. et al. (2014). A Fairness Load Balancing Algorithm in HWN Using a Multihoming Strategy, International Journal of Computers Communication \& Control, 9(5), 555-569, 2014.

[8] Dutta, S.; Mezzavilla, M.; Ford, R. et al. (2017). Frame Structure Design and Analysis for Millimeter Wave Cellular Systems, Wireless Communications IEEE Transactions, 16(3), $1508-1522,2017$.

[9] Ford, R.; Zhang, M.; Mezzavilla, M. et al. (2017). Achieving Ultra-Low Latency in 5G Millimeter Wave Cellular Networks, IEEE Communications Magazine, 55(3), 196-203, 2017.

[10] Gomez, K.; Goratti, L.; Granelli F. et al. (2014). A comparative study of scheduling disciplines in $5 \mathrm{G}$ systems for emergency communications, 1st International Conference on $5 \mathrm{G}$ for Ubiquitous Connectivity, 40-45, 2014. 
[11] Hassebo, A.; Muath Obaidat, M.; Ali M. A. (2018). Commercial 4G LTE cellular net works for supporting emerging IoT applications, 2018 Advances in Science and Engineering Technology International Conferences (ASET), IEEE, 1-6,2018.

[12] Ismail, S.; Ali, D. M.; Yosuf, A.L. (2019). MECC scheduling algorithm in vehicular environment for uplink transmission in LTE networks, International Journal of Electrical and Computer Engineering (IJECE), 9(2), 1191-1200, 2019.

[13] Jha, M.; Prateek, K.; Jaiswal, N. et al. (2016). Comparative Analysis of MAC Scheduling Algorithms in Long Term Evolution Networks using NS3, Asian Journal of Enginnering Technology and Innovation, 4(7), 124-127, 2016.

[14] Kawser, M. T.; Farid, H.M.A.B.; Hasin, A.R. et al. (2012). Performance Comparison between Round Robin and Proportional Fair Scheduling Methods for LTE, International Journal of Information and Electronics Engineering (IJIEE), 2(5), 2012.

[15] Marwan, A.A.; Perdana, D.; Sanjoyou, D.D. (2019). Performance Analysis of RAW Impact on IEEE 802.11ah Standard Affected by Doppler Effect, International Journal of Computers Communications \& Control, 14(2), 212-219, 2019.

[16] Mezzavilla, M.; Zhang, M.; Polese, M. et al. (2018). End-to-End Simulation of 5G mmWave Networks, Communications Surveys \& Tutorials IEEE , 20(3), 2237-2263, 2018.

[17] Niu, Y.; Li, Y.; Jin, D et al.(2015). A Survey of Millimeter Wave (mmWave) Communications for 5G: Opportunities and Challenges, . CoRR, abs/1502.07228.

[18] Perdana, D.; Dewanta,F.; Wibawa, I.P.D. (2017). Extending Monitoring Area of Production Plant Using Synchronized Relay Node Message Scheduling, 2017 Inter national, ournal of Communication Networks and Information Security, 20(3), 2237-2263, 2018.

[19] Putra, M.A.P.; Perdana D.; Negara, R.M. (2017). Performance Analysis of Data Traffic Offload Scheme on Long Term Evolution (LTE) and IEEE 802.11AH, Telekomnika, 15(4), 1659-1665, 2017.

[20] Rangan, S.; Rappaport, T. S.; Erkip, E. (2014). Millimeter-Wave Cellular Wireless Networks: Potentials and Challenges, Proceedings of the IEEE, 102, 366-385, 2014.

[21] Wulandari, T.; Perdana D.; Negara, R.M. (2018). Node Density Performance Analysis on IEEE 802.11ah Standard for VoIP Service, International Journal of Communication Networks and Information Security, 10(1), 2018. 